PHYSICAL REVIEW D 89, 063012 (2014)

\title{
Seedless clustering in all-sky searches for gravitational-wave transients
}

\author{
Eric Thrane ${ }^{1, *}$ and Michael Coughlin ${ }^{2}$ \\ ${ }^{1}$ LIGO Laboratory, California Institute of Technology, \\ MS 100-36, Pasadena, California 91125, USA \\ ${ }^{2}$ Department of Physics, Harvard University, Cambridge, Massachusetts 02138, USA
}

(Received 30 January 2014; published 25 March 2014)

\begin{abstract}
The problem of searching for unmodeled gravitational-wave bursts can be thought of as a pattern recognition problem: how to find statistically significant clusters in spectrograms of strain power when the precise signal morphology is unknown. In a previous publication, we showed how "seedless clustering" can be used to dramatically improve the sensitivity of searches for long-lived ( $10-1000 \mathrm{~s})$ gravitationalwave transients. To manage the computational costs, this initial analysis focused on externally triggered searches where the source location and emission time are both known to some degree of precision. In this paper, we show how the principle of seedless clustering can be extended to facilitate computationally feasible, all-sky searches where the direction and emission time of the source are entirely unknown. We further demonstrate that it is possible to achieve a considerable reduction in computation time by using graphical processor units, thereby facilitating more sensitive searches.
\end{abstract}

DOI: 10.1103/PhysRevD.89.063012

PACS numbers: 95.75.-z, 04.30.-w, 07.05.Bx

\section{INTRODUCTION}

Long-lived gravitational-wave transients (lasting $\sim 10-1000$ s) constitute an interesting class of signals for second-generation detectors such as Advanced LIGO [1] and Advanced Virgo [2]. After reaching design sensitivity, Advanced LIGO expects to observe $\approx 40$ binary neutron stars mergers and $\approx 10$ neutron-star black-hole coalescences per year of science data [3]. The standard searches for compact binary coalescences rely on matched filter template banks; see, e.g., [4,5]. More exotic sources of long-lived transients, including emission from rotational instabilities in protoneutron stars [6-9] and black-hole accretion disk instabilities [10-12], cannot be accurately modeled owing to theoretical uncertainties. However, searches for long-lived bursts [13-15] can be employed when a matched filter search is not possible. (There is a rich literature on short, subsecond gravitational-wave bursts and the different detection strategies available to detect them, but we focus here on long-lived transients.)

In a cross-correlation search such as [13,14], the detection of gravitational waves can be thought of as a pattern recognition problem. The goal is to find tracks of excess strain cross power, which appear as brighter-than-expected pixels on a signal-to-noise ratio spectrogram ( $f t$-map). In a previous work [15], we described how "seedless clustering" can be used to significantly enhance the sensitivity of searches for long-lived transients when a trusted matched filter template bank is not available. We review the details of seedless clustering in Sec. III, but the basic idea is to integrate along many different cleverly chosen paths in a

*ethrane@ligo.caltech.edu signal-to-noise ratio spectrogram. This is in contrast to seed-based clustering algorithms which form clusters from bright spectrogram pixels called "seeds."

The advantage of seedless clustering is most pronounced for long and weak signals [15]. For the waveforms considered in [15], we found that seedless clustering can detect a gravitational-wave signal (at a fixed false alarm and false dismissal rate) at a distance between $1.5 \times$ and $2 \times$ farther than a seed-based clustering algorithm. This corresponded to an increased detection volume of $4.2-7.4 \times$.

One of the challenges associated with seedless clustering is that it is, as a rule of thumb, more computationally expensive than seed-based alternatives. In [15], we focused on applications to targeted searches, in which the sky location is tightly constrained and the time of the event is known to exist in some "on-source" window, thereby saving the extra computational cost associated with searching many sky positions and emission times.

In this work, we show how the seedless clustering formalism from [15] can be extended to a high-sensitivity, computationally efficient, all-sky search for long-lived gravitational waves from arbitrary sky locations. There are two innovations which make this possible. First, by introducing a new random phase factor, we show that it is possible to efficiently scan the entire sky with a seedless clustering algorithm. Second, we take advantage of recent advances in computing to carry out our computations on graphical processor units (GPUs). Seedless clustering algorithms are "embarrassingly parallel" [16], which allows them to exploit the highly parallel architecture of GPUs. We show that an all-sky search with seedless clustering is both computationally feasible and more sensitive than 
a seed-based algorithm. We outline the computational requirements for a realistic search and demonstrate the advantage of carrying out computations on GPUs.

The remainder of the paper is organized as follows. In Sec. II, we describe some of the general features and challenges of an all-sky transient search. In Sec. III, we describe all-sky stochtrack, an all-sky algorithm which employs seedless clustering. In Sec. IV, we present the results of a sensitivity study comparing all-sky stochtrack to a seed-based algorithm. In Sec. V, we describe the computational resources required for realistic searches and compare the algorithms' performance on CPUs and GPUs. In Sec. VI, we offer concluding remarks and suggest directions for future research.

\section{THE CHALLENGES OF ALL-SKY RADIOMETRY}

In this section, we outline some of the general features of an all-sky transient search built on the principle of radiometry-using the time delay between two detectors to search data associated with a specific direction in the sky. We begin with strain time series $s_{I}\left(t^{\prime}\right)$ and $s_{J}\left(t^{\prime}\right)$ from detectors $I$ and $J$, which are separated by a displacement $\Delta \vec{x}$. (Note that $\Delta \vec{x}$ varies with time due to the rotation of the Earth.) The data are split into segments (typically with a duration of $\approx 1 \mathrm{~s}$ ) and Fourier transformed to create complex-valued strain spectrograms: $\tilde{s}_{I}(t ; f)$ and $\tilde{s}_{J}(t ; f)$. Note that $t^{\prime}$ refers to sampling time, whereas $t$ refers to segment start time.
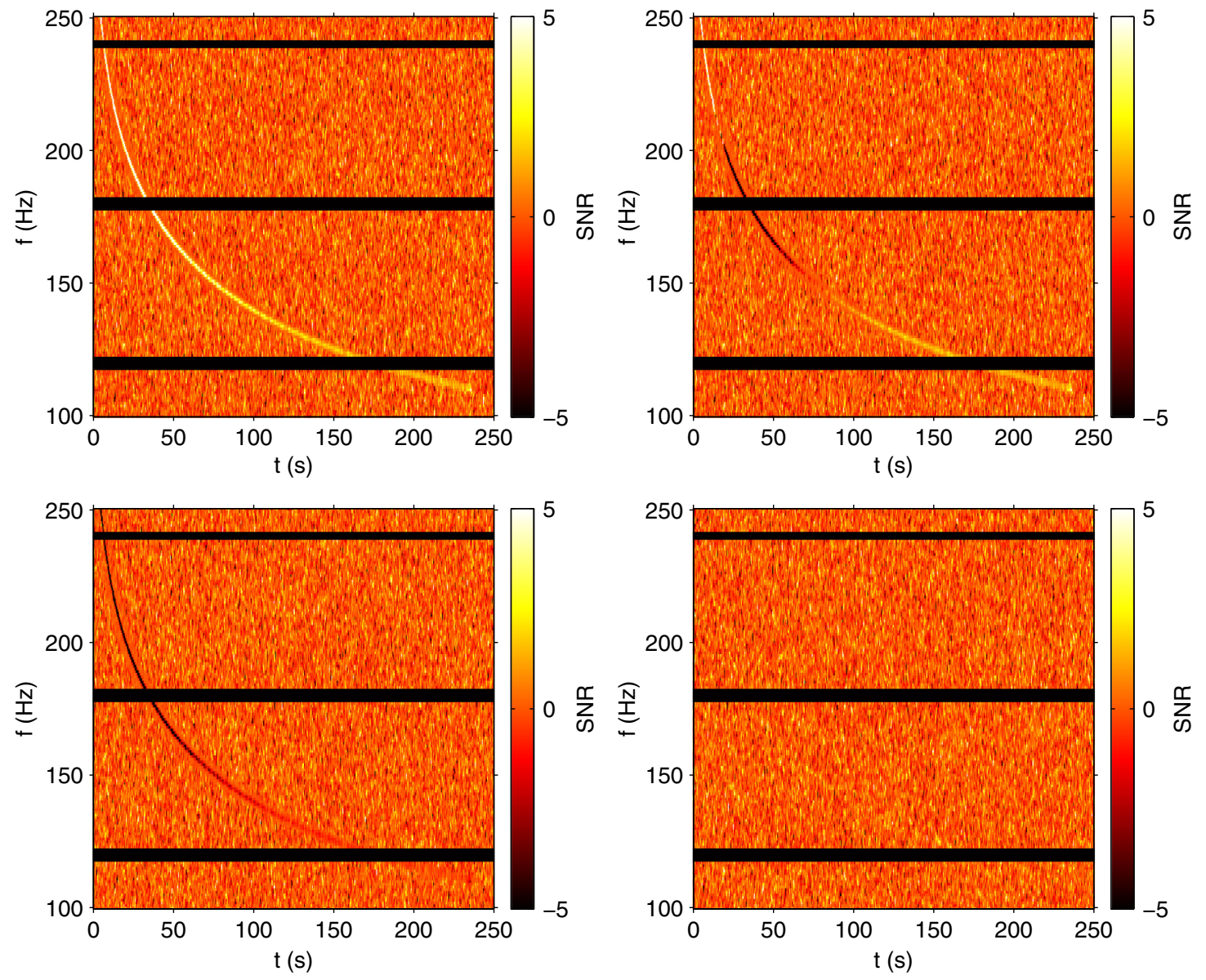

FIG. 1 (color online). The effect of filter mismatch. Top left: signal-to-noise ratio (SNR) spectrogram showing an accretion disk instability signal (ADI 2) in Advanced LIGO simulated Monte Carlo noise and obtained using the correct filter. The signal appears as a whitish-yellow track indicating positive SNR. The signal has been made very loud $(d=50 \mathrm{Mpc})$ for illustrative purposes. The black horizontal lines are notches due to instrumental artifacts. Top right: the same signal using an incorrect filter; i.e., the search direction does not match the source direction. The mismatch causes alternating stripes of positive (yellow) and negative (reddish black) SNR. At the turning points, where the SNR switches from positive to negative, the filtered cross-power signal is imaginary, and so the SNR (proportional to the real part of the filtered cross power) is approximately zero. Bottom left: the same signal using a different incorrect filter. In this case, the signal appears as purely negative. Bottom right: the same signal, using the correct filter, but much farther away $(D=340 \mathrm{Mpc})$. It is all but impossible to see the track with the naked eye, but it can nonetheless be detected by all-sky stochtrack with FAP $<0.1 \%$ without knowledge of the true direction or the signal morphology. 
Following [13-15], the signal-to-noise ratio spectrogram can be written as

$$
\rho(t ; f \mid \hat{\Omega})=\operatorname{Re}\left[\lambda(t ; f) e^{2 \pi i f \Delta \vec{x} \cdot \hat{\Omega} / c} \tilde{s}_{I}^{*}(t ; f) \tilde{s}_{J}(t ; f)\right] .
$$

Here, $e^{2 \pi i f \Delta \vec{x} \cdot \hat{\Omega} / c}$ is a phase factor, which takes into account the time delay between detectors $I$ and $J$ for a source located at $\hat{\Omega} ; c$ is the speed of light. The phase factor rotates the cross-power signal in the complex plane so as to be real and positive. The $\lambda(t ; f)$ term is a normalization factor, which uses neighboring segments to estimate the background [17]. Precise definitions of $\rho(t ; f \mid \hat{\Omega})$ and $\lambda(t ; f)$ are provided in Appendix A. If the source direction $\hat{\Omega}$ is known, for example, from an electromagnetic trigger (see [15]), then it is straightforward to apply to the appropriate phase factor. When no electromagnetic trigger is available, it is necessary to search over multiple directions.

Consider the case where the source is located at $\hat{\Omega}$ but the filter is chosen for the direction $\hat{\Omega}^{\prime}$, which introduces a timing error of

$$
\Delta \tau=\Delta \vec{x} \cdot\left(\hat{\Omega}-\hat{\Omega}^{\prime}\right) / c .
$$

On average, the timing error reduces the signal-to-noise ratio by

$$
R \equiv\left\langle\rho\left(t ; f \mid \hat{\Omega}^{\prime}\right)\right\rangle /\langle\rho(t ; f \mid \hat{\Omega})\rangle=\cos (2 \pi f \Delta \tau) \equiv \cos (\delta) .
$$

Inspecting Eq. (3), we can infer the qualitative features of a signal in a $\rho\left(t ; f \mid \hat{\Omega}^{\prime}\right)$ spectrogram characterized by a timing error $\Delta \tau$. For small values of $\delta$, the apparent signal will be weaker than it would in the absence of a timing error. This is because some of the cross power in Eq. (1) leaks into the imaginary direction. As $\delta$ crosses $\pi / 2$, the signal vanishes entirely before reappearing as a negative signal-tonoise ratio.

Graphically, large timing errors produce characteristic stripes in $\rho(t ; f \mid \hat{\Omega})$ spectrograms; see Fig. 1 . The bandwidth of each stripe is given by $1 / 4 \Delta \tau$. The minimum stripe size is $\Delta f_{\min }=1 / 4 \Delta \tau_{\max }$, where $\Delta \tau_{\max }$ is the travel time between detectors $I$ and $J$. For the two LIGO detectors, $\Delta f_{\min } \approx 25 \mathrm{~Hz}$.

We can define a tolerance for the maximum possible timing error by requiring that we observe no less than, say, $R=90 \%$ of the signal-to-noise ratio. It follows that

$$
\Delta \tau<\frac{1}{2 \pi f} \cos ^{-1}(R)
$$

As frequency increases, the tolerable timing error decreases. For signals in the most sensitive part of LIGO's band $f \approx 100 \mathrm{~Hz}$, the $R \geq 90 \%$ timing tolerance is $\Delta \tau \leq 720 \mu \mathrm{s}$. For high-frequency signals near $f \approx 1000 \mathrm{~Hz}$, it is $\Delta \tau \leq 72 \mu \mathrm{s}$ (corresponding to an angular error $\delta=26^{\circ}$ ).

To summarize, radiometry relies on the use of a phase factor to characterize the time delay between two detectors (a function of source sky position). If the assumed sky position is incorrect, gravitational-wave cross-power leaks from the positive real direction into imaginary and/or negative components, which, in turn, leads to a reduced signal-to-noise ratio. It may therefore be necessary to search many directions (with many time delays) in order to observe the signal with an acceptable signal-tonoise ratio.

\section{ALGORITHM}

In this section, we describe the details of an algorithm which uses seedless clustering to search for transient signals from all directions in the sky. We call it all-sky stochtrack. We begin with a brief review of the stochtrack algorithm [15], which will serve as a foundation on which to build.

The goal is to find the most significant cluster $\Gamma$ as determined by the value of the detection statistic [18],

$$
\mathrm{SNR}_{\mathrm{tot}} \equiv \frac{1}{N} \sum_{\{t ; f\} \in \Gamma} \rho(t ; f \mid \hat{\Omega}),
$$

where $\rho(t ; f \mid \hat{\Omega})$ is defined in Eq. (1) and $N$ is the number of pixels in $\Gamma$.

In any seedless clustering algorithm, $\Gamma$ is determined a priori by some set of rules (as opposed to by the data itself). In the stochtrack algorithm [15], $\Gamma$ is chosen randomly from the set of quadratic Bézier curves [19] subject to the constraint that the curve persists for a duration $t_{\min }$. (Other parametrizable curves such as spline can be used as well.) Each randomly selected Bézier curve is referred to as a "template." Each Bézier template is described by three time-frequency control points: $P_{0}$ $\left(t_{\text {start }}, f_{\text {start }}\right), P_{1}\left(t_{\text {mid }}, f_{\text {mid }}\right)$, and $P_{2}\left(t_{\text {end }}, f_{\text {end }}\right)$. The control points form a curve parametrized by $\xi=[0,1]$,

$$
\left(\begin{array}{c}
t(\xi) \\
f(\xi)
\end{array}\right)=(1-\xi)^{2} P_{0}+2(1-\xi) \xi P_{1}+\xi^{2} P_{2} .
$$

In order for the algorithm to have a high probability of guessing a close approximation to the true signal, many templates must be used. Fortunately, each template can be quickly generated from just six random numbers. By working with arrays of Bézier curves, stochtrack is able to carry out the sum in Eq. (5) for a large number of templates in parallel. As we shall see below, the parallel nature of the calculation lends itself to the use of GPUs.

The number of templates is denoted $T$. For practical applications, it is typically chosen to be $T=\mathcal{O}\left(10^{5}-10^{8}\right)$. In [15], we described a default search with $T=2 \times 10^{6}$ and 
a deep search (denoted stochtrack 10×) with $T=$ $2 \times 10^{7}$ [20].

The all-sky stochtrack algorithm builds on the foundation of stochtrack. First, we introduce "complex signal-tonoise ratio,"

$$
\mathfrak{p}(t ; f)=\lambda(t ; f) \tilde{s}_{I}^{*}(t ; f) \tilde{s}_{J}(t ; f)
$$

This is necessary to preserve the complex phase information that encodes the direction of the source. Note that, unlike $\rho(t ; f \mid \hat{\Omega}), \mathfrak{p}(t ; f)$ is not defined for a particular direction.

Next, in addition to the six random control points, we add an additional random variable $\Delta \tau$ corresponding to the time delay between the detectors, which, as we saw in Sec. II, is a proxy for sky location. Technically, $\Delta \tau$ is not a single number-for a fixed sky location, it slowly varies due to the rotation of the Earth. For the moment, however, we assume that $\Delta \tau$ is approximately constant over the duration of emission. We will return to this subtlety below. If we assume that the sky location of each transient is drawn from an approximately isotropic distribution, then the probability density function for time delay is a simple uniform distribution between $\pm \Delta \tau_{\text {max }}$.

Finally, we rewrite Eq. (5) to be

$$
\mathrm{SNR}_{\mathrm{tot}} \equiv \frac{1}{N} \sum_{\{t ; f\} \in \Gamma} \operatorname{Re}\left[e^{2 \pi i f \Delta \tau} \mathfrak{p}(t ; f)\right]
$$

The new sum described in Eq. (8) is carried out for many randomly selected clusters $\Gamma$, each with a randomly selected time delay $\Delta \tau$. By including a random time delay, the algorithm tries to guess not only the spectrographic shape of the signal but also the appropriate phase factor that will minimize the timing error stripes shown in Fig. 1. Minimizing the timing error maximizes $\mathrm{SNR}_{\mathrm{tot}}$.

The addition of a new random variable comes at a cost. First, the all-sky stochtrack algorithm will converge less quickly than stochtrack due to its expanded parameter space. Second, even if we imagine setting $T \rightarrow \infty$, all-sky stochtrack templates span a larger space than the templates used in stochtrack, and so all-sky stochtrack must contend with a comparatively higher background. That said, we find that the extra cost is small. In the next section, we show that, for several signal models, all-sky stochtrack achieves a sensitivity which is only slightly less than stochtrack, while searching a significantly expanded signal space.

In the discussion above, we assumed that the time delay $\Delta \tau$ can be approximated as constant over the duration of emission, even though it slowly varies due to the rotation of the Earth. We expect the rotation of the Earth to become increasingly important for longer signals and for higher frequency signals, which are more sensitive to timing errors. In practice, the method described here can be straightforwardly extended to accommodate the Earth's rotation. Instead of generating random time delays, one must generate random sky directions $\hat{\Omega}$ (chosen from an isotropic distribution), which can be used to calculate timevarying delay factors $\Delta \tau(t)$. However, for many signals, the constant $\Delta \tau$ approximation is adequate. We applied both methods to the waveforms considered here and observed no measurable improvement in sensitivity. If we imagine extending the current formalism to three or more detectors - beyond our present scope — then it will certainly be necessary to work with $\hat{\Omega}$ since each detector pair will have a different time delay.

\section{SENSITIVITY STUDY}

In this section, we describe a study to determine the sensitivity of all-sky stochtrack to four different long-lived test waveforms using simulated Monte Carlo and recolored LIGO noises. To compare with the baseline sensitivity of stochtrack, we use the same four waveforms used in [15]: two fallback accretion signals [6] abbreviated FA 1 and FA 2, and two accretion-disk instability waveforms [21], abbreviated ADI 1 and ADI 2. The waveforms span durations of 25-230 s and range in frequency from 110 to $1530 \mathrm{~Hz}$. Additional details about the waveforms and the parameters used to generate them are provided in Table IV of Appendix B. Additional information about the models behind the waveforms is available in [6] and [21]; see also [7-12].

Each waveform is injected into either Monte Carlo or recolored noise (initial LIGO noise which has been recolored to match the design sensitivity of Advanced LIGO while preserving nonstationary noise artifacts) [22]. The data are processed to form a complex signal-to-noise ratio spectrogram $\mathfrak{p}(t ; f)$ [see Eq. (7)]. Following [15], the ADI waveforms are analyzed in a band between 100 and $250 \mathrm{~Hz}$ while the FA waveforms are analyzed in a band between 700 and $1600 \mathrm{~Hz}$. The spectrogram resolution is $1 \mathrm{~s} \times 1 \mathrm{~Hz}$ except for FA 1 , for which we use $0.5 \mathrm{~s} \times 2 \mathrm{~Hz}$. Each spectrogram corresponds to $250 \mathrm{~s}$ of data [23]. Data segments are constructed with 50\%-overlapping Hann windows.

We characterize the sensitivity in terms of a detection distance, defined as the distance to which all-sky stochtrack can detect a source with a false-alarm probability FAP < $0.1 \%$ and a false-dismissal probability FDP $=50 \%$. We perform two series of tests. First, to compare all-sky stochtrack with stochtrack, we inject each signal with an optimal orientation (face on) and in an optimal sky location (where the detectors are most sensitive). The true source location is provided as input to stochtrack (and to the seedbased clustering algorithm, burstegard [24]), but the all-sky stochtrack algorithm is not provided any information about the true location of the source.

We show stochtrack results (from [15]) for the default search $\left(T=2 \times 10^{6}\right)$ and for the deep search $\left(T=2 \times 10^{7}\right)$, which is labeled stochtrack $10 \times$. We 
compare these to new results obtained with the default allsky stochtrack $\left(T=2 \times 10^{6}\right)$ and all-sky stochtrack $10 \times\left(T=2 \times 10^{7}\right)$.

In the second series of tests, we inject signals at random sky locations (ra, dec) (chosen from an isotropic distribution) and with random inclination and polarization angles $(l, \psi)$. We expect that the detection distance for signals recovered with random values of $(\mathrm{ra}, \mathrm{dec}, l, \psi)$ will be $\approx 60 \%$ of what is achieved for optimal sources based on the antenna response of our two-detector network.

TABLE I. Comparing all-sky stochtrack (all-sky seedless) sensitivity to stochtrack (tareted seedless) and burstegard (targeted seed-based) results from [15] using Monte Carlo noise. Both burstegard and stochtrack are provided the true sky location as an input, while all-sky stochtrack searches over the entire sky. By default, stochtrack and all-sky stochtrack perform $T=2 \times$ $10^{6}$ templates. The deep-search versions of stochtrack and all-sky stochtrack, denoted $10 \times$, use $T=2 \times 10^{7}$ templates. "Distance" refers to the distance at which a source is detected with false alarm probability $=0.1 \%$ and false dismissal probability $=50 \%$. We list both the absolute distance in Mpc and the percentage relative to the targeted seed-based algorithm. The ADI waveforms have been scaled assuming an energy budget of $E_{\mathrm{GW}}=0.1 M_{\odot}$. Volume is given in percent relative to the targeted seed-based algorithm. All the results are for optimally oriented sources in an optimal sky location except for entries marked "... w/random (ra, dec, $l, \psi)$," which represent the all-sky stochtrack results averaged over random sky locations and orientations.

\begin{tabular}{|c|c|c|c|c|}
\hline \multirow[b]{2}{*}{ Waveform } & \multirow[b]{2}{*}{ Algorithm } & \multicolumn{2}{|c|}{ Distance } & \multirow{2}{*}{$\begin{array}{c}\text { Volume } \\
\%\end{array}$} \\
\hline & & Absolute & $\%$ & \\
\hline \multirow[t]{6}{*}{ ADI 1} & Targeted seed-based & $370 \mathrm{Mpc}$ & 100 & 100 \\
\hline & Targeted seedless & $540 \mathrm{Mpc}$ & 150 & 320 \\
\hline & Targeted seedless $10 \times$ & $590 \mathrm{Mpc}$ & 160 & 420 \\
\hline & All-sky seedless & $490 \mathrm{Mpc}$ & 130 & 240 \\
\hline & All-sky seedless $10 \times$ & $540 \mathrm{Mpc}$ & 150 & 320 \\
\hline & w/random $(\mathrm{ra}, \mathrm{dec}, l, \psi)$ & $290 \mathrm{Mpc}$ & $\cdots$ & $\cdots$ \\
\hline \multirow[t]{6}{*}{ ADI 2} & Targeted seed-based & $190 \mathrm{Mpc}$ & 100 & 100 \\
\hline & Targeted seedless & $340 \mathrm{Mpc}$ & 180 & 560 \\
\hline & Targeted seedless $10 \times$ & $370 \mathrm{Mpc}$ & 200 & 740 \\
\hline & All-sky seedless & $310 \mathrm{Mpc}$ & 160 & 430 \\
\hline & All-sky seedless $10 \times$ & $340 \mathrm{Mpc}$ & 180 & 560 \\
\hline & $\ldots \mathrm{w} / \mathrm{random}(\mathrm{ra}, \mathrm{dec}, l, \psi)$ & $200 \mathrm{Mpc}$ & & $\cdots$ \\
\hline \multirow[t]{6}{*}{ FA 1} & Targeted seed-based & $17 \mathrm{Mpc}$ & 100 & 100 \\
\hline & Targeted seedless & $29 \mathrm{Mpc}$ & 150 & 320 \\
\hline & Targeted seedless $10 \times$ & $35 \mathrm{Mpc}$ & 180 & 560 \\
\hline & All-sky seedless & $22 \mathrm{Mpc}$ & 110 & 130 \\
\hline & All-sky seedless $10 \times$ & $24 \mathrm{Mpc}$ & 120 & 180 \\
\hline & ...w/random $(\mathrm{ra}, \mathrm{dec}, l, \psi)$ & $12 \mathrm{Mpc}$ & & $\cdots$ \\
\hline \multirow[t]{6}{*}{ FA 2} & Targeted seed-based & $25 \mathrm{Mpc}$ & 100 & 100 \\
\hline & Targeted seedless & $36 \mathrm{Mpc}$ & 150 & 320 \\
\hline & Targeted seedless $10 \times$ & $40 \mathrm{Mpc}$ & 160 & 420 \\
\hline & All-sky seedless & $30 \mathrm{Mpc}$ & 120 & 180 \\
\hline & All-sky seedless $10 \times$ & $36 \mathrm{Mpc}$ & 150 & 320 \\
\hline & $\ldots \mathrm{w} / \mathrm{random}(\mathrm{ra}, \mathrm{dec}, l, \psi)$ & $22 \mathrm{Mpc}$ & & $\cdots$ \\
\hline
\end{tabular}

Our hypothetical network consists of the Advanced LIGO detectors in Hanford, WA (H1) and Livingston, LA (L1) [1]. We assume both detectors are operating at design sensitivity.

The results of the study are summarized in Tables I and II for Monte Carlo and recolored noise, respectively. The Monte Carlo and recolored noise are processed identically except we apply a glitch identification [25] cut when analyzing recolored noise [26]. For optimally oriented sources injected into Monte Carlo noise, we find that the all-sky stochtrack $10 \times$ can see sources $120 \%-180 \%$ farther than the seed-based burstegard, even though the burstegard algorithm is given the known sky location, whereas all-sky stochtrack is not. This corresponds to an increased detection volume of $180 \%-560 \%$. For recolored noise, the improvement is $100 \%-180 \%$ in distance and $100 \%-$ $560 \%$ in volume.

Repeating the Monte Carlo analysis with the computationally cheaper default version of all-sky stochtrack $\left(T=2 \times 10^{7}\right)$, we obtain distances of $110 \%-160 \%$ times the distances obtained using burstegard. For recolored noise, these distances are $75 \%-160 \%$ times the values obtained using burstegard. Note that while burstegard can detect the FA 1 waveform in recolored noise at greater distances than the default version of all-sky stochtrack, this

TABLE II. The same as Table I except we utilize recolored noise from initial LIGO. An unphysical time shift is applied to spoil the coherence of any actual gravitational-wave signals that might have been present.

\begin{tabular}{|c|c|c|c|c|}
\hline \multirow[b]{2}{*}{ Waveform } & \multirow[b]{2}{*}{ Algorithm } & \multicolumn{2}{|c|}{ Distance } & \multirow{2}{*}{$\begin{array}{c}\text { Volume } \\
\%\end{array}$} \\
\hline & & Absolute & $\%$ & \\
\hline \multirow[t]{6}{*}{ ADI 1} & Targeted seed-based & $330 \mathrm{Mpc}$ & 100 & 100 \\
\hline & Targeted seedless & $540 \mathrm{Mpc}$ & 160 & 420 \\
\hline & Targeted seedless $10 \times$ & $540 \mathrm{Mpc}$ & 160 & 420 \\
\hline & All-sky seedless & $450 \mathrm{Mpc}$ & 130 & 240 \\
\hline & All-sky seedless $10 \times$ & $450 \mathrm{Mpc}$ & 130 & 240 \\
\hline & $\ldots \mathrm{w} / \mathrm{random}(\mathrm{ra}, \mathrm{dec}, l, \psi)$ & $280 \mathrm{Mpc}$ & $\cdots$ & $\cdots$ \\
\hline \multirow[t]{6}{*}{ ADI 2} & Targeted seed-based & $170 \mathrm{Mpc}$ & 100 & 100 \\
\hline & Targeted seedless & $310 \mathrm{Mpc}$ & 180 & 560 \\
\hline & Targeted seedless $10 \times$ & $340 \mathrm{Mpc}$ & 200 & 740 \\
\hline & All-sky seedless & $280 \mathrm{Mpc}$ & 160 & 420 \\
\hline & All-sky seedless $10 \times$ & $310 \mathrm{Mpc}$ & 180 & 560 \\
\hline & $\ldots \mathrm{w} / \mathrm{random}(\mathrm{ra}, \mathrm{dec}, l, \psi)$ & $210 \mathrm{Mpc}$ & $\cdots$ & $\cdots$ \\
\hline \multirow[t]{6}{*}{ FA 1} & Targeted seed-based & $22 \mathrm{Mpc}$ & 100 & 100 \\
\hline & Targeted seedless & $32 \mathrm{Mpc}$ & 150 & 320 \\
\hline & Targeted seedless $10 \times$ & $35 \mathrm{Mpc}$ & 160 & 420 \\
\hline & All-sky seedless & $16 \mathrm{Mpc}$ & 75 & 42 \\
\hline & All-sky seedless $10 \times$ & $22 \mathrm{Mpc}$ & 100 & 100 \\
\hline & $\ldots$ w/random $(\mathrm{ra}, \operatorname{dec}, l, \psi)$ & $11 \mathrm{Mpc}$ & $\cdots$ & $\cdots$ \\
\hline \multirow[t]{6}{*}{ FA 2} & Targeted seed-based & $25 \mathrm{Mpc}$ & 100 & 100 \\
\hline & Targeted seedless & $40 \mathrm{Mpc}$ & 160 & 420 \\
\hline & Targeted seedless $10 \times$ & $44 \mathrm{Mpc}$ & 180 & 560 \\
\hline & All-sky seedless & $30 \mathrm{Mpc}$ & 120 & 180 \\
\hline & All-sky seedless $10 \times$ & $33 \mathrm{Mpc}$ & 130 & 230 \\
\hline & $\ldots$ w/random $(\mathrm{ra}, \mathrm{dec}, l, \psi)$ & $21 \mathrm{Mpc}$ & $\cdots$ & $\cdots$ \\
\hline
\end{tabular}


is very likely because the burstegard algorithm is supplied with the true source location. In an apples-to-apples comparison, seedless clustering using the default stochtrack is more sensitive than burstegard [15].

Thus, the fact that burstegard can detect FA 1 signals in recolored noise at greater distances than all-sky stochtrack is telling us that it is very useful to know where in the sky to look when trying to find FA 1 waveforms in recolored noise. This is, perhaps, not surprising since the FA 1 waveform is shorter and spans a greater bandwidth than the other waveforms we consider. Shorter signals are more prone to resemble nonstationary noise. Signals with larger bandwidths are more prone to a loss of signal from a phase factor mismatch [see Eq. (8)].

We also present all-sky stochtrack detection distances for sources with random values of $(\mathrm{ra}, \mathrm{dec}, l, \psi)$, which are between $50 \%-68 \%$ of the values obtained for the case of an optimal source.

\section{COMPUTING}

The results from Sec. IV were obtained using graphical processor units (GPUs) on the LIGO Data Grid. In this section, we document how GPUs provide an efficient architecture for carrying out stochtrack and all-sky stochtrack calculations. We compare the performance of the algorithm using both GPUs and CPUs. We utilize Kepler GK104s GPUs, which are capable of peak single precision floating point performance of 4.6 Tflops according to the manufacturer. Each GPU card has 4 G memory. We use Intel Xeon E5-4650 CPUs.

For our benchmark test, we analyze spectrograms consisting of $151 \times 500$ pixels $(151 \mathrm{~Hz} \times 250 \mathrm{~s})$ using the same deep-search settings used to analyze the ADI 1 waveforms in the previous section. The computation time includes input-output tasks and other calculations, which do not take advantage of the GPU architecture. However, these computations correspond to a tiny fraction $(\lesssim 1 \%)$ of the total computation time. The results are summarized in Table III. We find that all-sky stochtrack calculations can be carried out $\approx 10 \times$ faster on GPUs than CPUs.

Using our benchmark tests, we estimate the computational requirements for full-fledged gravitational searches running stochtrack and all-sky stochtrack on GPUs. (Interestingly, stochtrack and all-sky stochtrack take about the same time to run given identical parameters.) We consider two analyses: one targeted (using stochtrack)

TABLE III. Relative computation times for all-sky stochtrack running on different architectures. The spectrogram is $151 \times 500$ pixels in size, and we use $T=2 \times 10^{7}$ templates.

\begin{tabular}{lc}
\hline \hline Hardware & Computation time \\
\hline CPU & $3800 \mathrm{~s}$ \\
GPU & $380 \mathrm{~s}$ \\
\hline \hline
\end{tabular}

and one all sky (using all-sky stochtrack). For both analyses, we assume an analysis band of $\Delta f=1200 \mathrm{~Hz}$ (following [13]). For the targeted analysis, we assume that the search analyzes $n_{\text {trig }}=50$ external triggers, e.g., from gamma-ray bursts; see [13]. Following [13], we assume that the search is carried out in a $\Delta t=1500 \mathrm{~s}$ wide onsource window. For the targeted analysis, we further assume that $n_{\mathrm{ts}}=100$ time-shift analyses are carried out in order to evaluate the significance of candidate events; see, e.g., [27]. For the all-sky analysis, we assume $n_{\mathrm{ts}}=10$.

Before we present estimates of computational cost, it will be useful to define a new variable: $T_{150}$, the number of templates per $150 \mathrm{~Hz}$ of bandwidth. This variable is useful since, all else equal, bigger bands must be analyzed with more templates than smaller bands due to the increased size of the template parameter space. We chose $150 \mathrm{~Hz}$ in order to facilitate comparisons with the ADI 1 and ADI 2 results given in Tables I and II. However, we note that waveforms FA 1 and FA 2 are analyzed in a $900 \mathrm{~Hz}$ wide band, 6 times wider than the ADI analysis band. Thus, $T_{150} \approx 3 \times 10^{5}$ corresponds to $T=2 \times 10^{6}$ (the default search) in the FA 1 and FA 2 analysis band. $T_{150}=2 \times 10^{7}$ corresponds to $\approx 1 \times 10^{8}$ (more sensitive than stochtrack $10 \times$ ) in the FA 1 and FA 2 analysis band.

Finally, we note that, all else equal, the number of templates required to maintain a fixed sensitivity increases linearly with frequency $T_{150} \propto f$ due to the increasing importance of timing error at higher frequencies; see Eq. (4). Indeed, it may be possible to improve the computational efficiency of the algorithm by preferentially choosing templates associated with higher frequencies.

Given our assumptions, the estimated computational time for a triggered stochtrack search with GPUs is

$$
\begin{aligned}
t_{c} \approx & 15 \text { days }\left(\frac{T_{150}}{2 \times 10^{7}}\right)\left(\frac{\Delta t}{1500 \mathrm{~s}}\right)\left(\frac{\Delta f}{1200 \mathrm{~Hz}}\right) \\
& \times\left(\frac{n_{\text {trig }}}{50}\right)\left(\frac{n_{\mathrm{ts}}}{100}\right)\left(\frac{128}{n_{\mathrm{GPU}}}\right) .
\end{aligned}
$$

Here $n_{\mathrm{GPU}}$ is the number of GPUs. The estimated computational time for an all-sky search with GPUs is

$$
\begin{aligned}
t_{c} \approx & 13 \text { days }\left(\frac{T_{150}}{3 \times 10^{5}}\right)\left(\frac{\Delta t}{1 \mathrm{yr}}\right)\left(\frac{\Delta f}{1200 \mathrm{~Hz}}\right) \\
& \times\left(\frac{n_{\mathrm{ts}}}{10}\right)\left(\frac{128}{n_{\mathrm{GPU}}}\right) .
\end{aligned}
$$

From Eq. (9), we conclude that GPUs can facilitate a deep-search sensitivity with stochtrack using modest computational resources. From Eq. (10), we conclude that a year-long all-sky analysis with default-sensitivity all-sky stochtrack can also be carried out using reasonable computational resources.

Since we know that $T_{150}=3 \times 10^{5}$ all-sky stochtrack sensitivity can improve significantly with added templates, 
it would be advisable to follow up on $\approx 10$ of the loudest events identified by the all-sky analysis, with a deeper $T_{150}=2 \times 10^{7}$ search. This would add only a marginal increase to the computational burden while ensuring that a marginal detection is promoted to a strong detection (or revealed to be a noise fluctuation).

The sensitivity of an all-sky search with all-sky stochtrack can be increased after the analysis has commenced (supposing, for example, that more GPUs become available) through the use of intermediate data files. Namely, we recommend recording $\mathrm{SNR}_{\mathrm{tot}}$ for each spectrogram. If multiple runs of the analysis are carried out, one can choose the largest value of $\mathrm{SNR}_{\text {tot }}$ among each run and for every spectrogram in a simple postprocessing step [28]. In other words, it is easy to combine the results from three runs with $T_{150}=3 \times 10^{5}$ in order to obtain results identical to a single $T_{150}=9 \times 10^{5}$ search. This parallelizability can be exploited to plan for a computationally conservative analysis, while being ready for a more aggressive analysis, should the resources be available.

\section{CONCLUSIONS AND FUTURE WORK}

In previous work, we proposed a new seedless clustering algorithm called stochtrack and demonstrated how it could significantly improve the sensitivity of searches for longlived, unmodeled gravitational-wave transients. Here we extend the principle of stochtrack to the case of an all-sky search, when there is no external trigger telling us where in the sky to look. We compare the sensitivity of all-sky stochtrack to that of a seed-based algorithm (which takes the true sky direction as input) and find that, for the most part, all-sky stochtrack is significantly more sensitive, even though it is searching for the signal in a much larger parameter space.
We point out that stochtrack and all-sky stochtrack are "embarrassingly parallel" algorithms, and we perform benchmark tests using CPUs and GPUs. We find that GPUs can carry out stochtrack and all-sky stochtrack calculations 10 times faster than CPUs. We estimate the computational cost of realistic analyses and show that interesting investigations can be carried out in a reasonable amount of time with a modest number of GPUs.

While we present all-sky stochtrack as a tool for all-sky analyses, it should also be very helpful in targeted analyses in which the sky localization of the external trigger is large compared to the point-spread function of the gravitationalwave detector network. Instead of drawing the time delay variable $\Delta \tau$ [see Eq. (8)] from a distribution derived from an isotropic prior, it is straightforward to draw it from a distribution corresponding to a particular patch of sky. This hybrid solution provides an efficient alternative to running stochtrack for many different directions.

We previously mentioned in [15] the possibility of using stochtrack to search for compact binaries. In general, compact binaries can be well modeled, and so it is expected that matched filtering is the optimal search strategy. However, the following are good reasons to explore alternative methods:

(i) Improve robustness and redundancy with an alternative method.

(ii) Investigate potentially challenging corners of parameter space, e.g., systems with non-negligible spin and/or eccentricity.

(iii) Detect exotic systems and/or new physics which are not included in matched filter template banks.

To place this discussion in context and to motivate future work, we close by reporting the results of a sensitivity study for detecting the coalescence of two $1.4 M_{\odot}$ neutron stars with stochtrack. We consider the case of an optimally
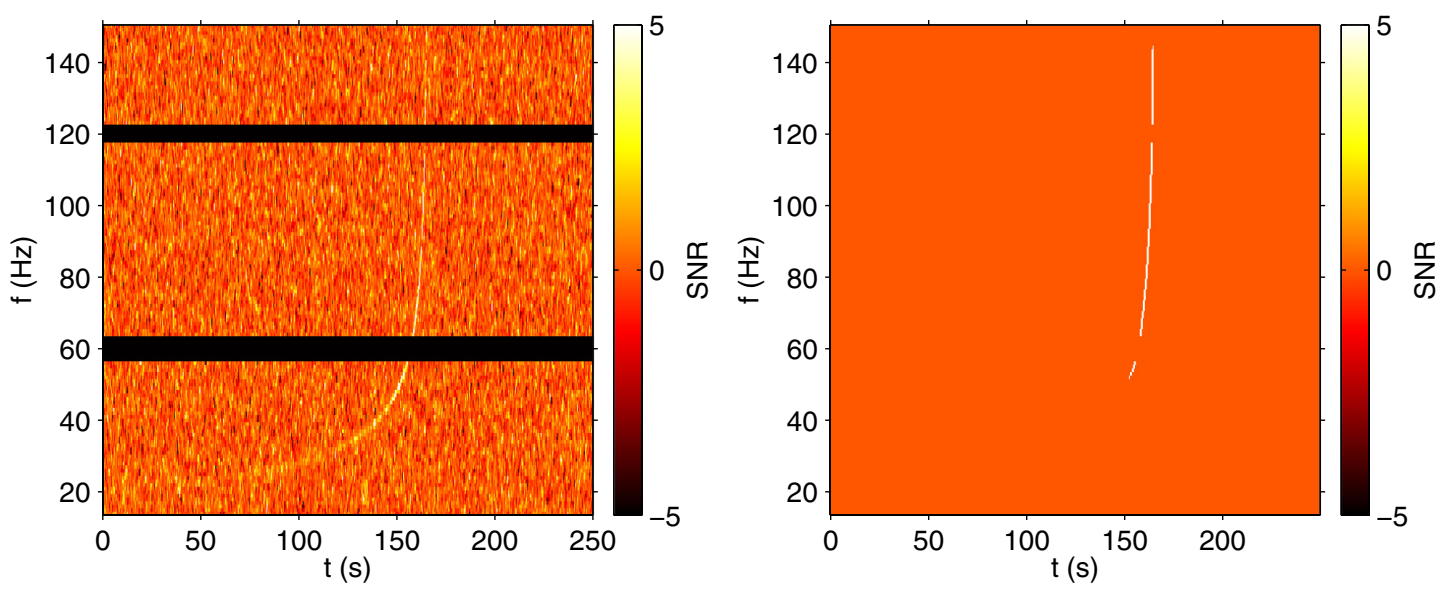

FIG. 2 (color online). Left: SNR spectrogram for a (very loud) binary neutron star signal in Monte Carlo noise. Right: the signal recovered by stochtrack. The stochtrack algorithm can detect binary neutron star signals in Advanced LIGO Monte Carlo noise with with FAP $=0.1 \%$ and FDP $=50 \%$ at a distance of $160 \mathrm{Mpc}$. Note that all-sky stochtrack does not do a good job of catching the beginning of the signal. This is not due to a lack of templates, but rather because the binary neutron star signal is not especially well described with a second-order Bézier curve. 
oriented system at an optimal sky location. We assume the signal is confined to a $660 \mathrm{~s}$ on-source region as in previous searches triggered by gamma-ray bursts [4].

We find that such a binary neutron star coalescence can be detected in Advanced LIGO Monte Carlo noise using stochtrack with FAP $=0.1 \%$ and FDP $=50 \%$ at a distance of $160 \mathrm{Mpc}$. By calculating the distance at which we can detect a face-on binary neutron star with FDP $=10 \%$ and $\mathrm{FAP}=50 \%$, we can estimate the value of a typical upper limit obtained from a nondetection. This projected upper limit distance is $150 \mathrm{Mpc}$. For comparison, the best $90 \%$ upper limit from initial LIGO and Virgo on binary neutron star coalescence coincident with gamma ray bursts is $37 \mathrm{Mpc}$ [4]. It is probable that the sensitivity of stochtrack to binary neutron stars can be enhanced with additional tuning; see Fig. 2. Thus, the application of stochtrack and all-sky stochtrack to compact binary coalescence signals appears promising and worthy of future work.

\section{ACKNOWLEDGMENTS}

We thank Stuart Anderson, Juan Barayoga, and Fred Donovan for assistance with GPUs. We thank Anthony Piro for sharing the fallback accretion waveforms used in this analysis. We thank Tanner Prestegard and Vuk Mandic for helpful comments on a draft of this paper. E. T. is a member of the LIGO Laboratory, supported by funding from United States National Science Foundation. LIGO was constructed by the California Institute of Technology and Massachusetts Institute of Technology with funding from the National Science Foundation and operates under Cooperative Agreement No. PHY-0757058. M. C. is supported by National Science Foundation Graduate Research Fellowship Program, under NSF Grant No. DGE 1144152.

\section{APPENDIX A: FORMALISM}

The signal-to-noise ratio spectrogram can be written as

$$
\rho(t ; f \mid \hat{\Omega})=\hat{Y}(t ; f \mid \hat{\Omega}) / \hat{\sigma}(t ; f \mid \hat{\Omega}) .
$$

TABLE IV. A summary of the waveforms used in our sensitivity study from [15]. The second and third columns describe the duration and frequency range of the waveform, respectively. The fourth column gives the spectrogram resolution used to analyze each waveform. The fifth column specifies the minimum signal duration assumed in each search. The ADI waveforms are downchirping accretion-disk instability waveforms [11,12,21], whereas the FA waveforms are up-chirping fallback accretion powered waveforms $[6,7]$.

\begin{tabular}{lrccr}
\hline \hline Waveform & Duration $(\mathrm{s})$ & $f_{\min }-f_{\max }(\mathrm{Hz})$ & $\delta t \times \delta f$ & $t_{\min }$ \\
\hline ADI 1 & 39 & $130-170$ & $1 \mathrm{~s} \times 1 \mathrm{~Hz}$ & $35 \mathrm{~s}$ \\
ADI 2 & 230 & $110-260$ & $1 \mathrm{~s} \times 1 \mathrm{~Hz}$ & $100 \mathrm{~s}$ \\
FA 1 & 25 & $1170-1530$ & $0.5 \mathrm{~s} \times 2 \mathrm{~Hz}$ & $20 \mathrm{~s}$ \\
FA 2 & 200 & $790-1080$ & $1 \mathrm{~s} \times 1 \mathrm{~Hz}$ & $100 \mathrm{~s}$ \\
\hline \hline
\end{tabular}

Here $\hat{Y}$ is an estimator for cross power,

$$
\hat{Y}(t ; f \mid \hat{\Omega})=\frac{2}{\mathcal{N}} \operatorname{Re}\left[Q_{I J}(t ; f \mid \hat{\Omega}) \tilde{s}_{I}^{*}(t ; f) \tilde{s}_{J}(t ; f)\right],
$$

and $\hat{\sigma}^{2}$ is an estimator for its variance,

$$
\hat{\sigma}^{2}(t ; f \mid \hat{\Omega})=\frac{1}{2}\left|Q_{I J}(t ; f \mid \hat{\Omega})\right|^{2} P_{I}^{\prime}(t ; f) P_{J}^{\prime}(t ; f) .
$$

Here $\mathcal{N}$ is the normalization from a discrete Fourier transform and $Q_{I J}(t ; f \mid \hat{\Omega})$ is a filter function, which accounts for the time delay between detectors $I$ and $J$ as well as the detector responses. Typically $Q_{I J}(t ; f \mid \hat{\Omega})$ is defined such that $\hat{Y}_{I J}(t ; f)$ is an unbiased estimator for gravitational-wave power [14]. The variables $P_{I}^{\prime}(t ; f)$ and $P_{J}^{\prime}(t ; f)$ are the autopower spectral densities for detectors $I$ and $J$ in the segments neighboring $t$.

It follows that

$$
\lambda(t ; f)=\frac{1}{\mathcal{N}} \sqrt{\frac{2}{P_{I}^{\prime}(t ; f) P_{J}^{\prime}(t ; f)}} .
$$

For additional details, the reader is referred to [14].

\section{APPENDIX B: MODEL PARAMETERS}

This section reproduces details about the test waveforms from [15]. The FA waveforms [6,7] are described by the following parameters: initial protoneutron star mass $M_{0}$, maximum neutron star mass $M_{\max }$, a dimensionless factor related to the supernovae explosion energy $\eta \approx 0.1-10$, and the radius of the protoneutron star $R_{0}$. The values of these parameters for FA 1 and FA 2 are given in Table V. The ADI waveforms [21] are parametrized by black hole mass $M_{\mathrm{BH}}$, dimensionless spin parameter $\alpha^{\star}=[0,1)$, the fraction of the accretion disk mask that forms clumps $\varepsilon \approx 0.01-0.2$, and the torus mass $m$. The values of these parameters for ADI 1 and ADI 2 are given in Table VI.

TABLE V. Parameters for FA waveforms from [15]. See [6] for additional details.

\begin{tabular}{lcccc}
\hline \hline Waveform & $M_{0}\left(M_{\odot}\right)$ & $M_{\max }\left(M_{\odot}\right)$ & $\eta$ & $R_{0}(\mathrm{~km})$ \\
\hline FA 1 & 1.3 & 2.5 & 10 & 20 \\
FA 2 & 1.3 & 2.5 & 1 & 25 \\
\hline \hline
\end{tabular}

TABLE VI. Parameters for ADI waveforms from [15]. See [21] for additional details.

\begin{tabular}{lcllc}
\hline \hline Waveform & $M_{\mathrm{BH}}\left(M_{\odot}\right)$ & $\alpha$ & $\epsilon$ & $m\left(M_{\odot}\right)$ \\
\hline ADI 1 & 5 & 0.3 & 0.05 & 1.5 \\
ADI 2 & 10 & 0.95 & 0.04 & 1.5 \\
\hline \hline
\end{tabular}


[1] G. M. Harry (for LIGO Scientific Collaboration), Classical Quantum Gravity 27, 084006 (2010).

[2] Virgo Collaboration, Advanced Virgo Baseline Design (2009), https://tds.ego-gw.it/itf/tds/file.php?callFile=VIR0027A-09.pdf.

[3] J. Abadie et al., Classical Quantum Gravity 27, 173001 (2010).

[4] J. Abadie et al., Astrophys. J. 760, 12 (2012).

[5] J. Abadie et al., Phys. Rev. D 85, 082002 (2012).

[6] A. L. Piro and E. Thrane, Astrophys. J. 761, 63 (2012).

[7] A. L. Piro and C. D. Ott, Astrophys. J. 736, 108 (2011).

[8] A. L. Piro and E. Pfahl, Astrophys. J. 658, 1173 (2007).

[9] A. Corsi and P. Mészáros, Astrophys. J. 702, 1171 (2009).

[10] K. Kiuchi, M. Shibata, P. J. Montero, and J. A. Font, Phys. Rev. Lett. 106, 251102 (2011).

[11] M. H. P. M. van Putten, Phys. Rev. Lett. 87, 091101 (2001).

[12] M. H. P. M. van Putten, Astrophys. J. Lett. 684, L91 (2008).

[13] J. Aasi et al., Phys. Rev. D 88, 122004 (2013).

[14] E. Thrane et al., Phys. Rev. D 83, 083004 (2011).

[15] E. Thrane and M. Coughlin, Phys. Rev. D 88, 083010 (2013).

[16] I. Foster, Designing and Building Parallel Programs: Concepts and Tools for Parallel Software Engineering (Addison-Wesley, Reading, MA, 1995).

[17] The $\lambda(t ; f)$ factor may also include a direction-dependent phase factor taking into account the relationship between the + and $\times$ polarizations of an elliptically polarized source. For the sake of simplicity, we do not include this additional phase factor. We expect the sensitivity to improve marginally by adding this phase factor by incorporating additional polarization information, though, at an increased computational cost.

[18] We note that alternative definitions of $\mathrm{SNR}_{\text {tot }}$ (with a different weighting scheme) are also possible; see [15].

[19] G. Farin, Curves and Surfaces for CAGD, Fourth Edition: A Practical Guide (Academic Press, New York, 1996).

[20] In [15], the default and deep search are said to use $T=$ $2 \times 10^{7}$ and $T=2 \times 10^{8}$, respectively. We believe the correct numbers are actually $T=2 \times 10^{6}$ and $T=2 \times 10^{7}$ as stated here.

[21] L. Santamaría and C. D. Ott, LIGO DCC No. T1100093, 2011, https://dcc.ligo.org/LIGO-T1100093-v2/public.

[22] The data are taken in between GPS times 822917487 and 847549782.

[23] A realistic search, using no external triggers to hone in on specific times, would require the analysis of significantly more data; typically about one year's worth. The calculations here use a small $250 \mathrm{~s}$ window in order to make direct comparisons with previous calculations [15]. The challenges of carrying out an analysis with a year's worth of data are described in Sec. V.

[24] T. Prestegard and E. Thrane, LIGO DCC No. L1200204, 2012, https://dcc.ligo.org/cgi-bin/DocDB/ShowDocument? docid=93146.

[25] T. Prestegard, E. Thrane, N. L. Christensen, M. W. Coughlin, B. Hubbert, S. Kandhasamy, E. MacAyeal, and V. Mandic, Classical Quantum Gravity 29, 095018 (2012).

[26] To apply the algorithm from [25], we assume that the source is optimally oriented with an optimal sky position.

[27] B. Abbott et al., Phys. Rev. D 69, 122001 (2004).

[28] Do not forget to use a different random seed for each run. 